\title{
Revisiting Binary Sequence Length Requirements for the Accurate Emulation of Highly Dispersive Transmission Systems
}

\author{
Jean-Christophe Antona ${ }^{1}$, Edouard Grellier ${ }^{1}$, Alberto Bononi ${ }^{2}$, Sébastien Petitrenaud ${ }^{1}$, Sébastien Bigo ${ }^{1}$
}

1 : Alcatel-Lucent, Bell-Labs, Route de Villejust, 91620 NOZAY - FRANCE.

2 : Dipartimento di Ingegneria dell' Informazione, Univ. di Parma, Viale G.P. Usberti, 43100, Parma, Italy Jean-Christophe.Antona @ alcatel-lucent.fr

\begin{abstract}
For accurate emulation, pseudo-random sequence lengths need not exceed requirements of MonteCarlo theory, scaling like the inverse of the expected bit error rate, even with strong inter-symbol correlations.
\end{abstract}

\section{Introduction}

When increasing channel bit rate beyond $10 \mathrm{~Gb} / \mathrm{s}$ or when operating over fibre lines with sparse or no inline dispersion compensation, the transmission regime changes into the so-called highly dispersive (or pseudo-linear) regime $[1,2]$. Thus, each pulse can expand over numerous neighbour pulses, and interact with them through optical non-linearities. For accurate emulation of these interactions, a few numerical studies recommend that Pseudo-Random Binary Sequences (PRBS) of sufficient length should be used [3, 4], with exponential dependence on bit rate and accumulated dispersion. For instance, with transmission of non-return-to-zero (NRZ) channels at $40 \mathrm{Gbit} / \mathrm{s}$ over $7 \times 100 \mathrm{~km}$ Standard Single Mode Fibre (SMF) without inline compensation, but optimized dispersion compensation at both ends, the required PRBS length should be as large as $2^{39}$ or $2^{52}$ bits, according to models from Ref. [4] or Ref. [3] respectively. Managing such lengths would not only take unrealistically long simulation times, but also raise serious concerns about the accuracy of lab measurements with bit-error rate (BER) test sets. However, the expected BERs generally lie between $10^{-2}$ and $10^{-6}$ before Forward Error-Correction (FEC), which suggests revisiting Monte-Carlo (MC)-like protocols to assess system performance. In such protocols, it is generally agreed that the required number of random noisy bits is about 100/BER [5], i.e. $10^{4}-10^{8}$ for a $10 \%$ relative error in absence of pulse-to-pulse interaction.

Next, we numerically estimate the required number of random, noisy bits using the MC method versus the expected BER, in highly dispersive systems where pulse-to-pulse interactions are high. Then, we determine the actual PRBS length that yields the same (sufficient) BER accuracy as the MC method.

\section{System under study}

In the following, the system under study consists of $7 \times 100 \mathrm{~km}$ SMF $(17 \mathrm{ps} / \mathrm{nm} / \mathrm{km}$ local chromatic dispersion) link with singly periodic dispersion maps similar to [6], for a single NRZ channel modulated at $43 \mathrm{~Gb} / \mathrm{s}$, with different values of residual dispersion per span between 0 (full inline compensation) and $1700 \mathrm{ps} / \mathrm{nm}$ (no inline compensation, most dispersive regime). For each configuration, we optimize pre- and post-dispersion compensation in the terminals, and vary the fibre input power, so as to get the power corresponding to $1.5 \mathrm{~dB}$ Optical Signal-to-Noise Ratio (OSNR) penalty at a reference BER (either $10^{-2}, 10^{-3}$ or $\left.10^{-5}\right)$. This power level, referred to as non-linear threshold (NLT) corresponds to a typical upper bound of operation in the non-linear regime. At the receiver end, noise is added voluntarily to reach the reference $\mathrm{BER}$. The receiver is modelled by $0.5 \mathrm{~nm}$-bandwidth $2^{\text {nd }}$ order Gaussian optical filter, followed by a photodiode, a $28 \mathrm{GHz}$ bandwidth $5^{\text {th }}$ order Bessel electrical filter and an ideal decision gate.

Contrary to PRBS methods associated with analytical BER estimation, Monte-Carlo simulations are achieved here by randomly drawing bit sequences and noise samples. The BER is estimated by error counting, once a decision threshold and a decision time are set. Let us consider a single-channel binary transmission system. The BER is the probability $p$ of getting an error on any bit. We define the indicator of the error event $\mathrm{l}_{\mathrm{j}}=1$ if an error occurs on the $\mathrm{j}$-th bit, or else $I_{j}=0$. Naturally, the expected value of $I_{j}$ is equal to p. In absence of correlations between the sampled signal and neighbouring bits, the number of noisy bits required to estimate a given $\mathrm{BER}$ with $10 \%$ relative error is about 100 /BER [5]. Here, we focus on systems where the samples have interacted and therefore become correlated across the transmission path. In this case, we explain our strategy for estimating the required number of noisy bits $\mathrm{N}$. In order to get $\mathrm{N}=\mathrm{LK}$ noisy bits, we perform $\mathrm{K}$ independent computation runs (thus $\mathrm{K}$ independent blocks) of $L$ bits each, with $L$ long enough with respect to the number of possibly interacting neighbours. For the investigated systems, values such as 256 bits are enough. For each block $k$ of $L$ bits, we then derive an estimator of the BER $\bar{p}_{L}(k)=\frac{1}{L} \sum_{j=1}^{L} I_{j}(k)$, and deduce the total Monte Carlo BER estimate as $\hat{p}_{N}=(1 / K) \sum_{k=1}^{K} \bar{p}_{L}(k)$. An unbiased estimation of the variance of $\hat{p}_{N}$ is $\hat{p}_{p}^{2}=\frac{1}{K\left(\begin{array}{ll}K & 1\end{array}\right)} \sum_{k=1}^{K}\left(\bar{p}_{L}(k) \quad \hat{p}_{N}\right)^{2}$. Finally, an estimation 
of the relative error is $\hat{}^{=}{ }_{p} / \hat{p}_{N}$. The Monte-Carlo iterative process consists in repeating new simulations of $L$ bits each until the estimation of the relative error becomes lower than $10 \%$.

To verify the reliability of the above procedure, we first performed simulations without inline dispersion compensation, at the NLT, i.e. $5.8 \mathrm{dBm}$, and $17.1 \mathrm{~dB}$ OSNR/0.1nm at the receiver. This ensures $\sim 10^{-3}$ BER after propagation. We ran the MC algorithm 100 times with different random seeds, and derived a standard deviation of BER estimates, of $0.810^{-4}$, and thus 0.08 error, just below $10 \%$, as shown in Fig. 1 . This confirms the validity of our stopping criterion on $\hat{\text {. }}$

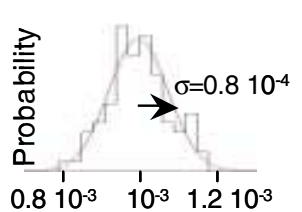

Bit-Error Rate

Figure 1: histogram of $\mathrm{BER}$ with $\mathrm{MC}$ runs

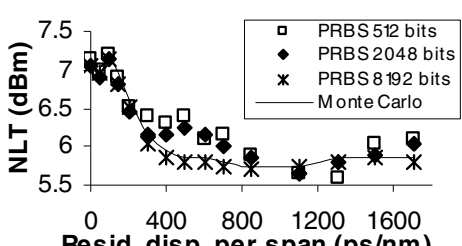

Resid. disp. per span (ps/nm)

Figure 2: NLT @ $10^{-3}$ BER for $\mathrm{MC}$ and PRBS simulations

\section{Required sequence length}

Next, we compare the requirements of the above MC method with the so-called PRBS method. Contrary to $\mathrm{MC}$, the PRBS method resorts to pre-defined bitsequence and uses semi-analytical BER estimation (provided by Karhunen-Loeve [7]). For the comparison, we first evaluate the number of bits required for an accurate estimation of the BER vs the residual dispersion per span (RDPS), i.e. the amount of inter-symbol correlation. Fig. 2 represents the NLT vs RDPS for MC simulations, and PRBS simulations with variable sequence lengths, for a reference BER of $10^{-3}$. Accurate $M C$ simulations are found to require $\sim 100 /$ BER whatever the RDPS. In contrast, the PRBS lengths required to provide the same accuracy as MC's are much smaller but should increase with RDPS. Otherwise, the NLT departs from MC predictions in Fig. 2. At this $10^{-3} \mathrm{BER}$, the PRBS length needs not exceed 8192 bits, despite strong inter-symbol interactions. This figure is well smaller than the predictions of theories of [3] and [4].

The work of Fig. 2 has been replicated at reference BERs $10^{-2}, 10^{-3}$ and $10^{-5}$ and the required sequence lengths derived. For PRBS simulations, the required length was estimated as the minimum length yielding the same BER as MC simulations. Fig. 3 summarizes the results. It depicts the evolution of the minimum required number of bits for $\mathrm{MC}$ and PRBS simulations versus the RDPS. One remarkable observation is that this number is found almost constant for $M C$ simulations and close to the expectation from [5], regardless of RDPS, and thus inter-symbol correlations. In contrast, when RDPS is relatively small, it can be seen that PRBS length requirements increase with RDPS, in accordance with [4], even though slightly smaller. At larger values of RDPS, when requirements from models [3] and [4] become comparable or higher to $\mathrm{MC}$ requirements for an expected BER, the PRBS length requirements prove to depend also on the expected BER, but stabilize to an asymptotic value which is smaller than $\mathrm{MC}$ requirements. For instance, with expected (but realistic, considering the use of FEC) BER of $10^{-3}$, no more than $2^{14}$ bits (16384) are actually required, no matter the dispersion regime, far from the $2^{39}-2^{52}$ bitspredictions of [3-4]. Indeed, the latter overlook the finite value of BER and that it needs not be estimated with less than $10 \%$ accuracy. It should be emphasized that this conclusion applies to typical preFEC BERs. From the above results, it can be extrapolated that BER estimates or measurements with at least $10 \%$ accuracy at $10^{-9}$ would require more than $2^{31}$ bit lengths in the highly dispersive regime.

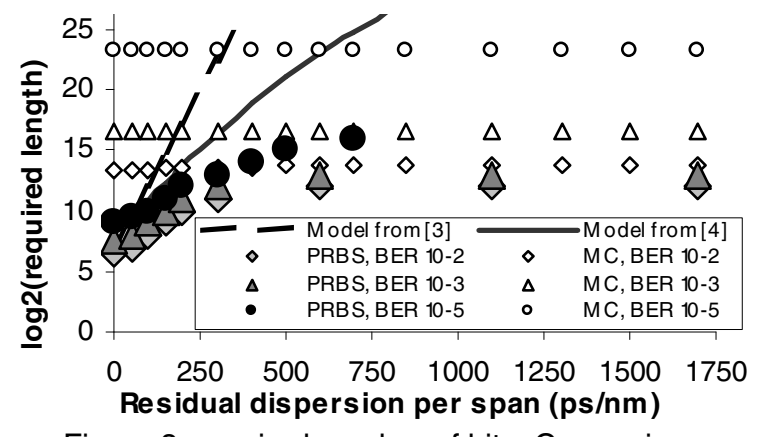

Figure 3: required number of bits. Comparison between models from [3-4] (dashed - solid line), and measured requirements at non-linear threshold for various reference BERs, using MC or PRBS methods.

\section{Conclusion}

We demonstrated that the number of bits needed to run $\mathrm{MC}$ simulations weakly depends on correlations between neighbouring bits in non-linear, highly dispersive transmission systems. MC theory was found to provide a reliable upper bound for sequence lengths in FEC-assisted, highly dispersive systems.

We acknowledge TCHATER project for funding.

\section{References:}

1. D. Van Den Borne et al, ECOC'06, Mo3.2.2, Cannes, Sept 2006

2. C. Laperle et al, OFC'07, PDP 16, Anaheim (2007)

3. L. K. Wickham et al., IEEE Photon. Technol. Lett., vol. 16 (2004), pp. 1591-1593.

4. P. Serena et al, ECOC'07, We.3.P093, Berlin, Sept. 2007.

5. G. Fishman, "Monte Carlo Concepts, Algorithms and Applications, NY, USA, Springer, $4^{\text {th }}$ ed., 2003.

6. J.-C. Antona et al, OFC'02, Anaheim, WX5, (2002)

7. E. Forestieri, J. Lightw. Technol., vol. 18, no. 11, pp. 1493-1503, Nov. 2000 